\title{
Multiple Brain Developmental Venous Anomalies as a Marker for Constitutional Mismatch Repair Deficiency Syndrome
}

\author{
(D) S.I. Shiran, DL. Ben-Sira, (DR. Elhasid, (D). Roth, DU. Tabori, (D) M. Yalon, (D) S. Constantini, and (D). Dvir
}

\begin{abstract}
BACKGROUND AND PURPOSE: Biallelic constitutional mutations in DNA mismatch repair genes cause a distinct syndrome, constitutional mismatch repair deficiency syndrome (CMMRD), characterized by cancers from multiple organs, most commonly brain tumors, during childhood. Surveillance protocols include total and brain MR imaging among other modalities to enable early detection of tumors. Brain surveillance scans revealed prominent brain developmental venous anomalies (DVAs) in some patients. DVAs are benign vascular anomalies, and their incidence in the general population is $2.6 \%-6.4 \%$. Most developmental venous anomalies are asymptomatic and are found incidentally. Our purpose was to assess the prevalence of DVAs in CMMRD patients and describe their phenotype.
\end{abstract}

MATERIALS AND METHODS: A retrospective descriptive analysis of brain MR imaging studies from 10 patients from 3 families with CMMRD was performed. Analysis included the number of developmental venous anomalies, location, draining vessels, and associated vascular anomalies (ie, cavernomas), with clinical correlation of symptoms and tumors.

RESULTS: All 10 patients had $\geq 2$ developmental venous anomalies, and 2 had, in addition, non-therapy-induced cavernomas. There was no clinically symptomatic intracranial bleeding from developmental venous anomalies. Six patients had malignant brain tumors. The location of brain tumors was not adjacent to the developmental venous anomalies. No new developmental venous anomalies developed during follow-up.

CONCLUSIONS: The occurrence of multiple developmental venous anomalies in all our patients with CMMRD suggests that developmental venous anomalies may be a characteristic of this syndrome that has not been previously described. If confirmed, this quantifiable feature can be added to the current scoring system and could result in early implementation of genetic testing and surveillance protocols, which can be life-saving for these patients.

ABBREVIATIONS: CMMRD = constitutional mismatch repair deficiency syndrome; DVA = developmental venous anomaly

onstitutional mismatch repair deficiency syndrome (CMMRD) is a cancer-predisposition syndrome characterized mainly by a high risk for developing cancer in childhood and

Received March 18, 2018; accepted after revision June 25

From the Departments of Radiology (S.I.S., L.B.-S.), Pediatric Hematology/Oncology (R.E., R.D.), and Pediatric Neurosurgery (J.R., S.C.), Dana Children's Hospital, Tel Aviv Sourasky Medical Center, Sackler Faculty of Medicine, Tel Aviv University, Tel Aviv, Israel; Division of Hematology/Oncology (U.T.), Department of Pediatrics, The Hospital for Sick Children, University of Toronto, Toronto, Ontario, Canada; and Department of Pediatric Hematology/Oncology (M.Y.), Sheba Medical Center, Tel-Hashomer, Israel.

Paper previously presented as a poster at: International Symposium on Pediatric Neuro-Oncology, June 28 to July 2, 2014; Singapore.

Please address correspondence to Shelly I. Shiran, MD, Department of Radiology, Dana Children's Hospital, Tel Aviv Sourasky Medical Center, Sackler Faculty of Medicine, Tel Aviv University, Tel Aviv, Israel, 6 Weizman St, Tel Aviv, 64239 Israel; e-mail: shellyshiran@gmail.com

三 Indicates article with supplemental on-line table.

http://dx.doi.org/10.3174/ajnr.A5766 young adulthood as well as nonmalignant features. The most common malignancies are brain tumors (predominantly malignant gliomas, though other tumors are reported), lymphoid malignancies (most commonly non-Hodgkins lymphomas and leukemia), and gastrointestinal cancers (Lynch syndrome-associated tumors, especially colorectal cancer). ${ }^{1,2}$ Nonmalignant manifestations of the syndrome include benign tumors such as adenomas and neurofibromas, features of neurofibromatosis type 1, predominantly café au lait macules, other hypo- and hyperpigmented skin alterations, pilomatricomas, and other features that were included in the suggested diagnostic criteria by the European Consortium. ${ }^{1}$

Because CMMRD is a cancer-predisposition syndrome with a very high penetrance and most reported individuals are affected during childhood, surveillance protocols were developed aiming at early detection and interventions of these cancers. MR imaging 
of the brain, every 6 months, starting at diagnosis or birth, is part of the surveillance protocol recommended for these children by the European Consortium Care for CMMR-D ${ }^{3}$ and the International Biallelic Mismatch Repair Deficiency Consortium. ${ }^{2,4}$ It is important to arrive at a definite diagnosis of CMMRD in a pediatric or young adult patient with cancer as early as possible to allow the recommended surveillance for the patient and genetic counseling for family members. To facilitate CMMRD testing in patients with cancer, the European Consortium Care for CMMR-D suggested a score based on diagnostic criteria. ${ }^{1}$ In the suggested score, several features of the syndrome are assigned points; a patient with a 3-point score or above is referred for genetic counseling. In Tel Aviv Sourasky medical center, we noted prominent developmental venous anomalies (DVAs) on routine brain MR imaging performed as a part of our surveillance protocol in patients with CMMRD.

DVA is the most frequently encountered cerebral vascular malformation, with an incidence of $2.6 \%-6.4 \%$ in different studies. ${ }^{5,6}$ A DVA is characterized by a cluster of venous radicles that converge into a collecting vein, resulting in the typical caput medusa appearance of the DVA. ${ }^{7}$ The collecting vein crosses a variable length of brain parenchyma to join either the superficial or deep venous system. Two or more DVAs coexisting in separate regions of the brain were observed in $7 \%-16 \%$ of described patients with DVAs. ${ }^{8,9}$ Most DVAs are asymptomatic and are found incidentally. However, DVAs have been documented as a rare cause of cerebrovascular bleeding and ischemic events. ${ }^{7}$ There is a known association between DVAs and cavernomas. ${ }^{6,7}$

Our aim was to assess the prevalence of DVAs in children with CMMRD and to describe their phenotype to elucidate possible distinct features associated with CMMRD.

\section{MATERIALS AND METHODS}

We performed detailed clinical and imaging analysis of all children from 3 different families that are carriers of DNA mismatch repair genes, each family with a distinct type of mutation. The first family has a PMS2 mutation (c.2458dupA), with 5 affected homozygous members. The second family has a MSH6 mutation [c.2314c $>$ T (p.Arg772Trp)], with 2 affected homozygous members. The third family has 2 different pathogenic mutations in MSH6 (c.3984_3987dupGTCA//c.3959_3962delCAAG), with 3 affected members who are compound heterozygotes.

All children had surveillance brain MR imaging during the past 10 years. This retrospective study was approved by Tel Aviv Sourasky medical center institutional review board. A retrospective descriptive analysis of their brain MR imaging studies included the number of DVAs, location, draining vessels (peripheral or central), length and diameter of the collecting vein, associated parenchymal changes (as reflected by increased T2WI signal intensity), and the presence of associated cavernomas. Correlation with coexisting brain tumor presence and location was also performed. Descriptive statistics were performed with SPSS (IBM, Armonk, New York).

\section{RESULTS}

Brain MR imaging studies of 10 children with CMMRD were evaluated. The complete patient information is presented in the
On-line Table. There were 6 boys and 4 girls. The age at the earliest study available per child ranged from 1.0 to 12.0 years (mean, 6.5 years). Seven children were treated for CNS tumors (4 glioblastoma multiforme, 1 medulloblastoma, 1 gliomatosis cerebri, and 1 cord primitive neuroectodermal tumor), 1 child was treated for acute myeloid leukemia (AML), and 2 children were treated for lymphoma. Colon polyposis was present in 6 children, and adenocarcinoma of the colon, in 1 child. DVAs were noted in all children, constituting $100 \%$ prevalence. Five children had 2 DVAs, 1 child had 3 DVAs (Fig 1), 3 children had 5 DVAs, and 1 child had 7 DVAs. In total, 35 DVAs were observed; $83 \%$ were in the supratentorial brain and $64 \%$ drained to peripheral cortical veins. The diameters of the collecting veins ranged from a minimum of $0.8 \mathrm{~mm}$ to a maximum of $3.0 \mathrm{~mm}$ (average, $1.9 \mathrm{~mm}$; median, $1.8 \mathrm{~mm}$; mode, $2.2 \mathrm{~mm}$ ). The length of the collecting vein ranged from a minimum of $5.0 \mathrm{~mm}$ to a maximum of $39.0 \mathrm{~mm}$ (average, $24.9 \mathrm{~mm}$; median, $27.0 \mathrm{~mm}$; mode, $30.0 \mathrm{~mm}$ ). There were $\mathrm{T} 2$ signal changes in the brain parenchyma adjacent to the DVA in $26 \%$ of DVAs (Fig 2).

In children with brain tumors, there was no correlation between the tumor locations and the location of the DVA. Cavernomas were present in 2 patients. One patient with a brain tumor had a single small cavernoma adjacent to the DVA before therapy; on follow-up studies, after tumor treatment with radiation therapy, he developed focal bleeding in the known cavernoma and bleeding in additional therapy-induced multiple cavernomas that were not apparent on the initial study. A second patient had 2 non-therapy-induced cavernomas present but distant from the DVA. For 9 children, follow-up studies were available; the time between first and last available studies ranged from 0.2 to 7.5 years, and there was no change in the number of DVAs on follow-up studies.

\section{DISCUSSION}

In this study, we observed $100 \%$ prevalence of multiple DVAs in children with CMMRD. This is strikingly different from the prevalence of DVAs in the healthy population, which was reported as $2.7 \%$ in a study based on postmortem evaluation, ${ }^{5}$ and $6.4 \%$ in a more recent study based on modern MR imaging. ${ }^{6}$ Gökçe et al ${ }^{6}$ evaluated 1165 patients with brain MR imaging studies and found DVAs in 75 patients; of those, 10 had multiple DVAs, which is $0.9 \%$ of their total evaluated population and $13.3 \%$ of the group of patients who had DVAs. The phenotypic characteristics of the DVAs in their study included 73\% supratentorial location, 50\% drainage to peripheral veins, and a collecting vein diameter range of $1.0-4.3 \mathrm{~mm}$ (median, $2.0 \mathrm{~mm}$ ). These findings are not significantly different from ours with $83 \%$ supratentorial location, $64 \%$ drainage to peripheral veins, and a collecting vein diameter range of $0.8-3.0 \mathrm{~mm}$ (median, $1.8 \mathrm{~mm}$ ). We found associated T2 signal changes in the parenchyma adjacent to the DVA in $26 \%$ of the DVAs, which is within the range of those reported in different studies: $7.8 \%,{ }^{10} 11.6 \%,{ }^{8}$ and $28 \% .{ }^{11}$ The phenotypic similarities of the DVAs in our study group compared with those reported in the general population exclude morphologic unique features of this vascular malformation in CMMRD patients.

An increased prevalence of DVAs was reported in association with extensive head and neck venolymphatic malformations. In a 


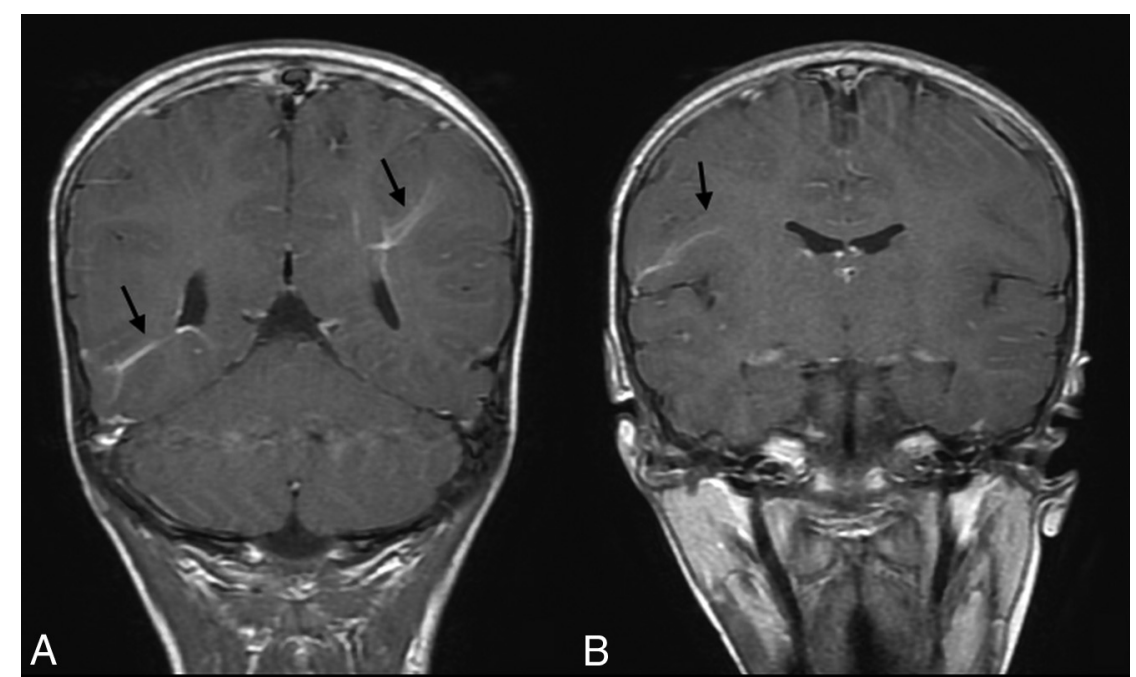

FIG 1. Coronal T1-weighted postgadolinium injection images ( $A$ and $B$ ) demonstrate 3 prominent DVAs (arrows) in a girl who is homozygote for the PMS2 mutation.

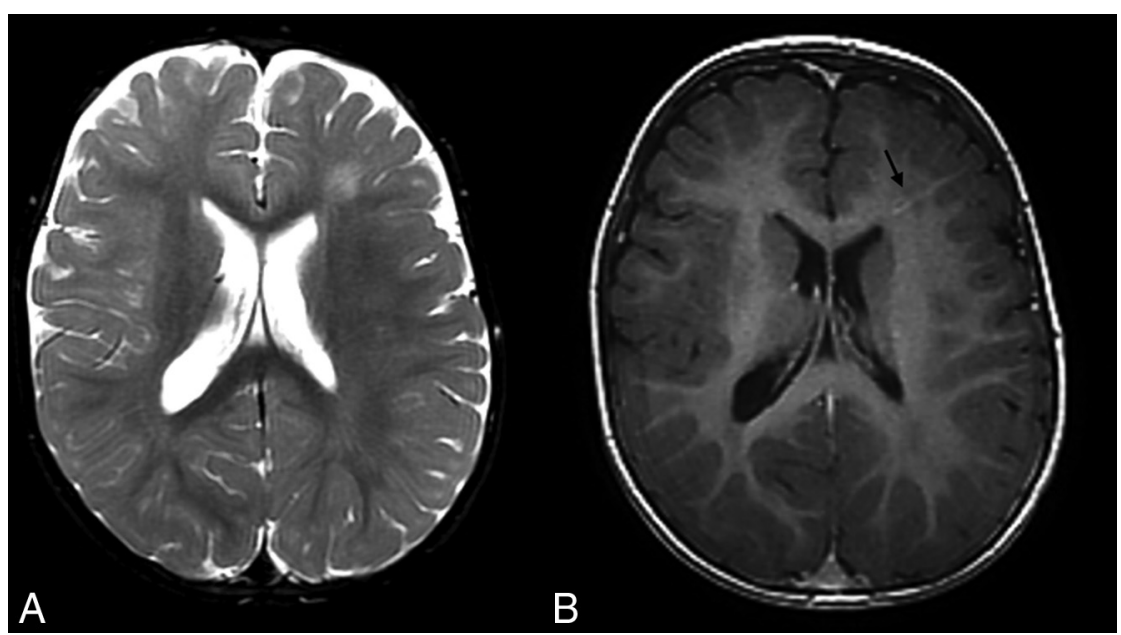

FIG 2. MR imaging study of a boy who is homozygote for the PMS2 mutation and has 2 DVAs demonstrates increased T2 signal in the left frontal white matter on axial T2WI (A). This is associated with a subtle DVA (arrow) depicted in the axial TIWI postgadolinium injection (B).

series of 40 patients with facial venous malformations, $20 \%$ had associated DVAs and $12.5 \%$ had multiple DVAs. ${ }^{12}$ In a series of 33 patients with orbital and periorbital lymphatic or venolymphatic malformations, up to $60 \%$ of patients had associated DVAs. ${ }^{13} \mathrm{We}$ did not encounter associated head and neck vascular malformations in our study population, and we did not find an association between CMMRD and vascular malformations in the literature. It has been suggested that DVAs may be part of intracranial manifestations of neurocutaneous disorders because there are case reports describing an association between DVAs and blue rubber bleb nevus syndrome. ${ }^{14,15}$ Patients with CMMRD have not been reported to have any association with blue rubber bleb nevus syndrome, but there is a known phenotypic overlap with neurofibromatosis type 1 , most commonly café-au-lait macules. ${ }^{16}$ However, there are no known reports describing the prevalence of DVAs in patients with neurofibromatosis type 1. From our experience (unpublished data), the prevalence of DVAs in the neurofibromatosis type 1 population is not as high as that seen in this study. Baas et $\mathrm{al}^{17}$ reported an association between corpus callosum agenesis, gray matter heterotopia, and CMMRD; these findings were not present in our study group.

Jones et $\mathrm{al}^{18}$ reported an increased prevalence of DVAs in a population of patients with primary brain tumors compared with the healthy population; in their study, $10.2 \%$ of patients with brain tumors had a DVA present compared with $5.3 \%$ of the control group. Among patients with DVAs, only 4 had $>1$ DVA. They did not find any correlation between the tumor and DVA locations, which is consistent with our results of no correlation between tumor site and DVA location. In their study, they did not mention whether children with CMMRD were included among the patients with brain tumors. Among studies describing patients with CMMRD, in a French cohort of 31 patients, ${ }^{19}$ there was mention of only 2 patients with intracranial angiomas; assuming the term "angioma" represents a DVA, this finding is very different from the prevalence in our study. In the French cohort, the description of intracranial abnormalities is based on patients' physician reports and not on dedicated evaluation of brain MR imaging by a neuroradiologist. Because a DVA may be considered a normal variant, its mention may be omitted from radiology reports; this may explain the discrepancy. Most studies describing patients with CMMRD are dedicated to the clinical course and genetics; we did not find, to date, an additional description of associated intracranial vascular malformations.

In a child with a tumor, clinical suspicion of CMMRD arises when coexistence of several features is observed. These include a consanguineous family, café au-lait spots (possibly mimicking neurofibromatosis type 1), and a family history of malignancies in young relatives, especially brain tumors, lymphomas, or leukemias. Indeed, these and additional clinical manifestations were recently suggested to serve as criteria for a clinical diagnosis of CMMRD. ${ }^{1}$ Our data suggest that the presence of multiple DVAs in an MR imaging of the brain with an associated brain tumor or other CMMRD-related malignancy can be another clue to the diagnosis. This marker can be especially helpful to determine the clinical diagnosis of CMMRD in individuals who are unaffected by cancer and in whom the genetic tests are inconclusive. Most interesting, in 2 patients, in whom there was clinical suspicion of CMMRD, the lack of DVAs was associated with negative molecular evidence of CMMRD (unpublished data).

The main limitation of this study is the small group of patients. 
Further assessment of larger patient cohorts from the international consortia will further delineate the exact prevalence of DVAs and the characteristics of DVAs in this patient population. However, the presence of multiple DVAs in all patients in this study has no parallel description in any other pathology, supporting the important role of DVAs for the clinical diagnosis of CMMRD.

\section{CONCLUSIONS}

Our cohort had $100 \%$ prevalence of multiple CNS DVAs in patients with CMMRD. If confirmed, this quantifiable feature can be added to the current scoring system and result in early implementation of genetic testing and surveillance protocols, which can be life-saving for these patients and families as well as allowing targeted tumor management. ${ }^{20,21}$

\section{ACKNOWLEDGMENTS}

We thank the International Biallelic Mismatch Repair Deficiency Consortium for their support in this work. This research was further supported by Meagan's Walk, the CIHR Joint CanadaIsrael Health Research Program, and an American Association for Cancer Research Stand Up 2 Cancer Catalyst TM Research Grant.

\section{REFERENCES}

1. Wimmer K, Kratz CP, Vasen HFA, et al; EU-Consortium Care for CMMRD (C4CMMRD). Diagnostic criteria for constitutional mismatch repair deficiency syndrome: suggestions of the European consortium 'Care for CMMRD' (C4CMMRD). J Med Genet 2014;51: 355-65 CrossRef Medline

2. Tabori U, Hansford JR, Achatz MI, et al. Clinical management and tumor surveillance recommendations of inherited mismatch repair deficiency in childhood. Clin Cancer Res 2017;23: e32-37 CrossRef Medline

3. Vasen HF, Ghorbanoghli Z, Bourdeaut F, et al; EU-Consortium Care for CMMR-D (C4CMMR-D. Guidelines for surveillance of individuals with constitutional mismatch repair-deficiency proposed by the European Consortium "Care for CMMR-D" (C4CMMR-D). J Med Genet 2014;51:283-93 CrossRef Medline

4. Bakry D, Aronson M, Durno C, et al. Genetic and clinical determinants of constitutional mismatch repair deficiency syndrome: report from the constitutional mismatch repair deficiency consortium. Eur J Cancer 2014;50:987-96 CrossRef Medline

5. Sarwar M, McCormick WF. Intracerebral venous angioma: case report and review. Arch Neurol 1978;35:323-25 CrossRef Medline

6. Gökçe E, Acu B, Beyhan M, et al. Magnetic resonance imaging findings of developmental venous anomalies. Clin Neuroradiol 2014;24: 135-43 CrossRef Medline
7. Ruız DS, Yilmaz H, Gailloud P. Cerebral developmental venous anomalies: current concepts. Ann Neurol 2009;66:271-83 CrossRef Medline

8. Linscott LL, Leach JL, Zhang B, et al. Brain parenchymal signal abnormalities associated with developmental venous anomalies in children and young adults. AJNR Am J Neuroradiol 2014;35: 1600-07 CrossRef Medline

9. Lee C, Pennington MA, Kenney CM. MR evaluation of developmental venous anomalies: medullary venous anatomy of venous angiomas. AJNR Am J Neuroradiol 1996;17:61-70 Medline

10. Santucci GM, Leach JL, Ying J, et al. Brain parenchymal signal abnormalities associated with developmental venous anomalies: detailed MR imaging assessment. AJNR Am J Neuroradiol 2008;29: 1317-23 CrossRef Medline

11. San Millan Ruíz D, Delavelle J, Yilmaz H, et al. Parenchymal abnormalities associated with developmental venous anomalies. Neuroradiology 2007;49:987-95 CrossRef Medline

12. Boukobza M, Enjolras O, Guichard JP, et al. Cerebral developmental venous anomalies associated with head and neck venous malformations. AJNR Am J Neuroradiol 1996;17:987-94 Medline

13. Bisdorff A, Mulliken JB, Carrico J, et al. Intracranial vascular anomalies in patients with periorbital lymphatic and lymphaticovenous malformations. AJNR Am J Neuroradiol 2007;28: 335-41 Medline

14. Gabikian P, Clatterbuck RE, Gailloud P, et al. Developmental venous anomalies and sinus pericranii in the blue rubber-bleb nevus syndrome: case report. J Neurosurg 2003;99:409-11 CrossRef Medline

15. Chung JI, Alvarez H, Lasjaunias P. Multifocal cerebral venous malformations and associated developmental venous anomalies in a case of blue rubber bleb nevus syndrome. Interv Neuroradiol 2003; 9:169-76 CrossRef Medline

16. Wimmer K, Rosenbaum T, Messiaen L. Connections between constitutional mismatch repair deficiency syndrome and neurofibromatosis type 1. Clin Genet 2017;91:507-19 CrossRef Medline

17. Baas AF, Gabbett M, Rimac M, et al. Agenesis of corpus callosum and gray matter heterotopia in three patients with constitutional mismatch repair deficiency syndrome. Eur J Hum Genet 2013;21:55-61 CrossRef Medline

18. Jones BV, Linscott L, Koberlein G, et al. Increased prevalence of developmental venous anomalies in children with intracranial neoplasms. AJNR Am J Neuroradiol 2015;36:1782-85 CrossRef Medline

19. Lavoine N, Colas C, Muleris M, et al. Constitutional mismatch repair deficiency syndrome: clinical description in a French cohort. J Med Genet 2015;52:770-78 CrossRef Medline

20. Bouffet E, Larouche V, Campbell BB, et al. Immune checkpoint inhibition for hypermutant glioblastoma multiforme resulting from germline biallelic mismatch repair deficiency. J Clin Oncol 2016;34: 2206-11 CrossRef Medline

21. Kline CN, Joseph NM, Grenert JP, et al. Targeted next-generation sequencing of pediatric neuro-oncology patients improves diagnosis, identifies pathogenic germline mutations, and directs targeted therapy. Neuro Oncol 2017;19:699-709 CrossRef Medline 